\title{
Guy Jobert, Exister au travail. Les hommes du nucléaire
}

Toulouse, Érès, coll. « Clinique du travail », 2014

\section{Marie Ponnet}

\section{OpenEdition}

\section{Journals}

Édition électronique

URL : http://journals.openedition.org/travailemploi/7321

DOI : $10.4000 /$ travailemploi.7321

ISSN : 1775-416X

Éditeur

DARES - Ministère du Travail

Édition imprimée

Date de publication : 1 octobre 2016

Pagination : 110-114

ISSN : 0224-4365

Référence électronique

Marie Ponnet, «Guy Jobert, Exister au travail. Les hommes du nucléaire », Travail et Emploi [En ligne], 148 | octobre-décembre 2016, mis en ligne le 11 juillet 2019, consulté le 21 décembre 2020. URL: http://journals.openedition.org/travailemploi/7321 ; DOI : https://doi.org/10.4000/travailemploi.7321 


\section{Exister au travail. Les hommes du nucléaire}

\section{Guy Jobert}

Toulouse, Érès, coll. « Clinique du travail », 2014, 407 p.

\section{Lu par Marie Ponnet"}

Cet ouvrage, publié en 2014, présente les résultats d'une recherche-intervention menée vingt ans plus tôt par l'auteur, sociologue, auprès d'agents de conduite en centrale nucléaire. Alors qu'une réforme de l'organisation du travail (baptisée par la direction « démarche conduite ») est en cours de mise en œuvre sur l'ensemble du parc nucléaire français, l'encadrement de l'une des centrales demande à Guy Jobert d'effectuer « le "suivi" local de la "démarche-conduite" » (p. 25). L'objectif est de découvrir si « les changements prévus sont effectifs» (p. 27). Ceux-ci concernent le travail des agents de conduite qui, « présents en permanence », pilotent les réacteurs et ont donc « le monopole des interventions humaines sur la machine en fonctionnement » (p. 31), ce qui leur confère une grande autonomie professionnelle et leur vaut le surnom de « seigneurs de la conduite » (p. 33). Or leur travail, souvent peu spectaculaire lorsqu'ils l'exercent en salle de commande, est mystérieux, voire invisible, aussi bien pour la direction que pour les observateurs extérieurs comme G. Jobert. À l'issue d'une phase de familiarisation avec son terrain, l'auteur découvre que les agents de conduite ont non seulement la réputation de se plaindre mais se plaignent effectivement régulièrement, si bien que « la question de départ de la recherche, l'énigme à résoudre, devient alors la plainte et sa signification » (p. 43).

Pour ce faire, la démarche de l'auteur entend « ne jamais considérer séparément les sujets, leur agir et leurs contextes de vie et d'action, contextes toujours peuplés d'humains et d'objets techniques » (p. 10). En ce sens, G. Jobert prévient le lecteur que son approche souhaitait au départ privilégier les situations de travail, et non le travail. Pourtant, cet objet s'est imposé en raison de son importance dans la construction identitaire des individus.

L'auteur rappelle que la souffrance au travail est toujours aussi prégnante dans notre société. Réalité cinglante, elle a également été mise en lumière par la sociologie du travail $\left(\right.$ Linhart $^{1}$, Gollac et Volkoff ${ }^{2}$ ). Le phénomène est d'autant plus important

\footnotetext{
* Chercheuse associée au Centre nantais de sociologie (Cens, UMR 6025), université de Nantes.

1. Linhart D. (2010), La Modernisation des entreprises, $3^{\mathrm{e}}$ éd., Paris, La Découverte.

2. Gollac M., Volkoff S. (1996), « Citius, altius, fortius. L'intensification du travail », Actes de la recherche en sciences sociales, $\mathrm{n}^{\circ} 114$, pp. 54-67.
} 
qu'il ne concerne plus seulement les travailleurs traditionnellement « dominés », mais s'étend à des catégories sociales qui en étaient jusque-là préservées : ce que Stéphane Beaud et Marie Cartier ont appelé «la déstabilisation des stables ${ }^{3}$ ». Moins classiquement, le dessein de l'auteur n'est pas seulement de discuter avec la communauté scientifique, mais d'apporter aux lecteurs non chercheurs des « outils » sous forme de « théories et concepts » (p. 16) et de démontrer la richesse de l'apport des sciences humaines et sociales sur la compréhension des phénomènes sociaux.

En entrant sur le terrain par la plainte, qu'il définit comme résultant « du déséquilibre du bilan rétribution-contribution » (p. 20) subjectivement établi par les agents de conduite, l'auteur rappelle dès l'introduction son cadre d'analyse. Considérant comme Hannah Arendt que les possibilités de subjectivation des actes de travail ne suffisent pas à expliquer la motivation des agents au travail, il fait l'hypothèse que cette plainte exprime une souffrance et que sa permanence crée une expérience douloureuse de leur condition au travail, qui a pour origine l'absence de reconnaissance par autrui de leur activité. Or cette reconnaissance est nécessaire à la construction de l'identité professionnelle.

Dans le premier des cinq chapitres, l'auteur décrit le terrain, la problématique et la méthodologie de son enquête. Il revient sur le passage du «problème social » au «problème sociologique » (p. 25) en mettant au cœur de sa démarche, nous l'avons dit, « la plainte » des agents de conduite et non les conséquences des nouvelles organisations du travail, comme le souhaitaient pourtant les commanditaires. Selon lui, pour qu'une posture de recherche soit possible, plusieurs conditions doivent être réunies. Le commanditaire doit notamment laisser au chercheur la possibilité de se livrer à une contextualisation élargie du problème posé en fonction des approches théoriques de sa discipline. Les impératifs épistémologiques et méthodologiques doivent primer sur «l'urgence de l'action ou sur la contingence politique ou économique » (p. 32). Tout chercheur qui, un jour ou l'autre, a travaillé sur un sujet dans le cadre d'une relation contractuelle trouvera sûrement ici un écho à ses propres pratiques ${ }^{4}$. Véritable œuvre de mise en visibilité du cadre de la recherche, ce chapitre donne à voir une riche réflexion épistémologique.

Dans le deuxième chapitre, théorique, G. Jobert présente le cadre conceptuel qu'il mobilise dans la suite de l'ouvrage pour interpréter les faits recueillis au cours de sa recherche-action. Il présente ici le statut qu'il donne aux individus qu'il a rencontrés, et s'attache à montrer comment ces agents doivent innover et inventer des solutions pour atteindre les objectifs qui leur sont fixés ou résoudre un problème qui dépasse les consignes. Pour cela, l'auteur s'appuie non seulement sur des travaux de psychodynamique du travail (Christophe Dejours et Yves Clot) et de psychanalyse (Sigmund

3. Beaud S., Cartier M. (2006), « De la précarisation de l'emploi à la précarisation du travail. La nouvelle condition salariale », in Beaud S., Confavreux J., Lindgaard J. (dir.), La France invisible, Paris, La Découverte, pp. 561-573. 4. Notamment le décalage qui existe entre la perception de l'étude par la population étudiée et par les commanditaires (cf. Flamant N. [2005], « Observer, analyser, restituer. Conditions et contradictions de l'enquête ethnologique en entreprise », Terrain, $\mathrm{n}^{\circ} 44$, pp. 137-152). 
Freud), mais aussi sur des travaux de philosophie (Paul Ricœur) pour montrer en quoi les liens entre « Ego, réel et autrui » (p. 93 et suivantes) tissent la trame de l'intersubjectivité. La mobilisation de diverses approches disciplinaires rend l'explication relative à l'obtention de la reconnaissance au travail difficile d'accès : le cheminement emprunté par l'auteur est parfois aride, si bien que des liens logiques peuvent assez aisément échapper au lecteur profane.

Dans le chapitre suivant, G. Jobert teste l'hypothèse selon laquelle la plainte des agents de conduite « exprime une dimension douloureuse du rapport subjectif au travail » (p. 110), qui provient d'un manque de reconnaissance. Présent dans de nombreux univers de travail, ce manque est particulièrement important dans le monde hyperprocéduralisé, complexe et à risques des centrales nucléaires. Analysant l'activité de travail des agents de conduite, l'auteur y distingue deux sortes de contributions : celle qui est explicite et formalisée comme le travail prescrit (décrit dans la fiche de poste) ou les contraintes de quart (horaires de nuit, pénibilité liée à la rotation des postes), et celle qui, « indicible » (p. 112), a à voir avec la charge psychique du pilotage d'une installation nucléaire (p. 122) et comprend donc la peur, l'anxiété et l'angoisse présentes au quotidien. La contribution réelle des agents de conduite se caractérise par l'ingéniosité dont ils font preuve dans leur travail. Loin d'être un pur et simple « travail d'exécution » des consignes dans un système rigide, le travail des agents de conduite mobilise un savoir-faire issu de leur expérience du métier ${ }^{5}$, qui peut aller jusqu'à la transgression des normes. Paradoxalement, cette «tricherie » (p. 124) de même que leurs « inventions » leur permettent de se conformer aux objectifs de production et de sécurité. Or la hiérarchie ne peut pas valoriser de telles pratiques, sauf à remettre en cause les normes techniques établies et l'organisation du travail.

Guy Jobert s'interroge alors sur le « bilan contribution-rétribution » de ces agents, en rapportant leur rétribution monétaire (salaire, primes, heures supplémentaires) à leur travail effectif. Il note que la charge psychique de l'activité ne fait pas l'objet d'une rétribution en tant que telle, pas plus que l'ingéniosité au travail, la peur, l'anxiété ou l'angoisse : cette forme de contribution des agents n'étant pas identifiée, sa reconnaissance devient de fait impossible. L'auteur fait ainsi «l'hypothèse que cette reconnaissance impossible constitue la source majeure de la plainte répétitive exprimée par les agents » (p. 189). Nous regrettons toutefois que cette « plainte», omniprésente et centrale dans la problématique suivie, ne soit ni mise en mots par les acteurs euxmêmes, ni visible dans la description de leur quotidien, ni véritablement qualifiée.

Dans le quatrième chapitre, la question centrale que pose l'auteur est de savoir si les agents de conduite trouvent dans leur activité la reconnaissance que le rapport salarial ne leur donne pas. Cette absence de reconnaissance symbolique de la contribution intellectuelle et psychique n'est jamais présente dans les discours car elle est

5. Dans le secteur du bâtiment et des travaux publics, Nicolas Jounin (2006) montre très bien ce phénomène : « La sécurité au travail accaparée par les directions. Quand les ouvriers du bâtiment affrontent clandestinement le danger », Actes de la recherche en sciences sociales, $\mathrm{n}^{\circ}$ 165, pp. 72-91. 
refoulée. Pour interroger le rapport des agents à leur activité concrète et aux objets techniques, l'auteur emprunte à $\mathrm{H}$. Arendt la distinction qu'elle introduit dans son ouvrage Condition de l'homme moderne (1994) entre travail, œuvre et action. Selon G. Jobert, « la seule façon pour les agents de voir leur travail devenir action passe par l'accomplissement d'actes contraires aux normes techniques ou aux règles de métier. C'est à cette condition qu'ils peuvent accéder à des arènes de jugement qui dépassent le cercle restreint des collègues les plus proches » (pp. 212-213). Le paradoxe est que la reconnaissance au travail ne vient pas seulement de l'excellence dans le faire mais aussi, et même surtout, dans le fait de parvenir à faire valoir aux yeux d'autrui la réussite de l'activité dans ces systèmes à risques.

L'auteur souligne que les agents de conduite, peu diplômés, ont un salaire de base assez faible. Ce sont finalement les primes et les heures supplémentaires ${ }^{6}$ qui, augmentant leurs revenus, leur permettent une ascension sociale et parfois aussi une mise en couple avec une femme au statut social plus élevé que le leur. L'analyse de l'origine sociale des agents de conduite est ici menée de manière particulièrement fine et intéressante. S'appuyant sur les travaux de Claude Dubar ${ }^{7}$, qui montrent que la construction de l'identité passe notamment par la référence à la profession ou au métier, G. Jobert explique que la situation des agents de conduite ne peut s'inscrire dans ce cadre. En effet, leur activité qui consiste à « conduire » et à « surveiller » une installation automatisée n' offre pas la possibilité de constituer un « référentiel professionnel [suffisamment] fort » (p. 285) et parlant au-delà des murs de l'entreprise. Pour pouvoir tout de même se construire une identité, les agents de conduite se réfèrent donc à « une communauté de condition de vie » (p. 285), celle du travail en rythme décalé.

Dans le dernier chapitre, l'auteur se demande alors : "Pourquoi les agents donnent-ils au-delà de l'obligation que leur impose la relation salariale contractuelle ? » (p. 304). Pour répondre à cette question, G. Jobert s'appuie sur l' « essai sur le $\mathrm{don}^{8} »$ de Marcel Mauss. Il en conserve surtout «l'obligation de donner » (p. 305), soulignant que la contribution des agents de conduite est d'assumer pour le compte de la société une activité porteuse de vie et de mort et qui, de fait, ne peut pas trouver de reconnaissance en termes de réciprocité. Selon l'auteur, les agents de conduite ont à la fois une dette vis-à-vis de leur employeur (puisque leurs conditions salariales leur ont permis une ascension sociale) et un double devoir par rapport à la société (fournir l'énergie et garantir la sûreté nucléaire). Cette dette « originaire » (p. 335), qu'ils ont contractée vis-à-vis de l'employeur qui leur a donné la vie en leur donnant la possibilité d' « opérer une véritable transmutation de leur capital initial » (p. 337)

6. Représentant jusqu'à $80 \%$ de suppléments de salaire.

7. Dubar C. (1991), La Socialisation. Construction des identités sociales et professionnelles, Paris, Armand Colin ; Dubar C. (1992), «Formes identitaires et socialisation professionnelle », Revue française de sociologie, vol. 33, $\mathrm{n}^{\circ} 4$, pp. 505-529.

8. Mauss M. (1973), «Essai sur le don. Forme et raison de l'échange dans les sociétés archaïques », in Sociologie et anthropologie, $3^{\mathrm{e}}$ éd., Paris, Presses universitaires de France ; introduction de Claude Lévi-Strauss. Article originalement publié dans L'Année sociologique, seconde série, 1923-1924. 
via leur ascension sociale, doit être acquittée par un contre-don qui consiste à donner une part d'eux-mêmes au-delà de l'obligation salariale contractuelle.

Dans cet ouvrage, G. Jobert réussit plusieurs paris. Le premier est d'avoir réalisé un important travail épistémologique et conceptuel. Le deuxième est d'avoir puisé des références à la fois dans la philosophie, la sociologie, la psychologie, la psychanalyse ou encore l'économie, dont les données ne souffrent pas d'obsolescence et dont la diversité permet un regard renouvelé sur le travail. Le troisième est d'avoir consacré une étude approfondie et originale ${ }^{9}$ aux agents de conduite des centrales nucléaires, qui n'avaient jusqu'alors fait l'objet que de très peu d'enquêtes alors même que l'objet « souffrance au travail » ou, plus largement, celui des conséquences des formes d'organisation du travail sur la santé, a, lui, connu de nombreux développements. L'entrée par la «plainte» des agents, ainsi que les explications avancées sur son origine et ses causes, nous semble inédite. Enfin, cet ouvrage particulièrement agréable à lire s'adresse à la communauté scientifique mais aussi à toute personne intéressée par les sciences humaines et sociales, ou curieuse d'en savoir plus sur ces travailleurs du nucléaire.

9. Par, répétons-le, la mobilisation de la philosophie et de la psychanalyse. 